\title{
Meteor Storms as a Window on the Delivery of Extraterrestrial Organic Matter to the Early Earth
}

\author{
P. Jenniskens \\ The SETI Institute, 2035 Landings Drive, Mountain View, CA 94043, \\ $U S A$
}

\begin{abstract}
The unique rarefied flow and flash heating in meteors creates physical conditions that can change exogenous organic matter into unique prebiotic molecules. With the exception of rare giant comet impacts, most infalling matter at the time of the origin of life was deposited in the atmosphere during the meteor phase. Much new data has been obtained from observations in the Leonid Multi-Instrument Aircraft Campaign; a series of NASA and USAF sponsored Astrobiology missions that explored the 1998-2002 Leonid meteor storms. Here, we provide an overview of some of this recent insight, which provides a framework in which the prebiotic chemistry can be studied.
\end{abstract}

\section{Introduction}

Meteors are of interest to the origin of life debate because they represent a potential important pathway from exogenous carbon to prebiotic compounds (Oró 1961; Chyba \& Sagan 1992; Chyba \& Sagan 1997). While meteorites and interplanetary dust particles provide convenient laboratory samples to study the properties of the organic matter in space, most of that organic matter is ablated in the atmosphere during a phase called a "meteor", and irrevocably altered. Perhaps conveniently, because some enrichment in oxygen, for example, could greatly increase the usefulness of the exogenous organic matter for prebiotic processes (Jenniskens et al. 2000a). These products will continue to interact with the Earth's atmosphere and ultimately settle to Earth's surface. Before addressing the atmospheric interactions, it is necessary to understand the physics and chemistry of the meteor phase itself.

Unfortunately, products of this organic chemistry are now rapidly diluted in the Earth's bio mass. Much of our understanding of this organic chemistry will have to come from a better understanding the physical conditions in meteors (Jenniskens 2001). The interaction of small meteoroids with the Earth's atmosphere is still a poorly understood problem, despite much previous research (Öpik 1958; Levin 1961; McKinley 1961; Bronsthen 1983; Ceplecha et al. 1998). The fate of organic matter in the meteors remains particularly hard to address because organic molecules are so difficult to detect by remote sensing. Atomic carbon has no strong optical emissions in the visible and near-IR. Small molecules such as $\mathrm{CN}, \mathrm{CH}$ and $\mathrm{C}_{2}$ can be detected more readily at optical wavelengths, but no certain detections have been made to date. Any more complex 


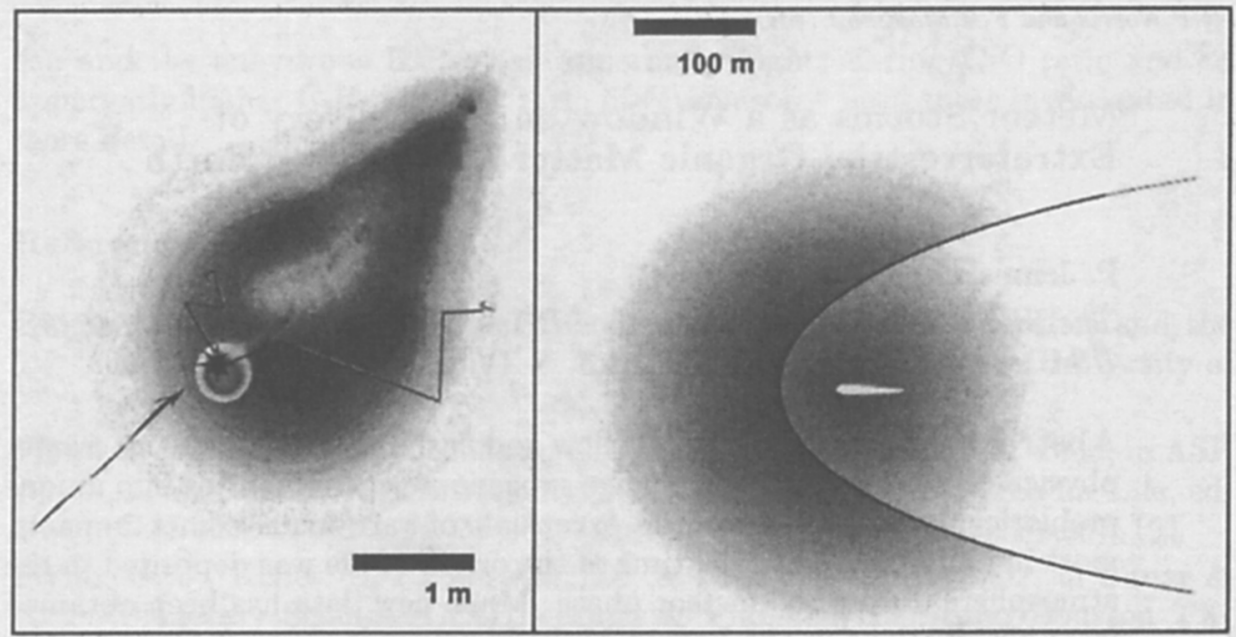

Figure 1. Popular depiction of meteor morphology. Left: air molecules hit vapor cloud products to expand into $\sim 2 \mathrm{~m}$ diameter air plasma volume. Right: UV light generated in this process is absorbed in ambient atmosphere, causing an optical glow and "shock".

molecule will be present at low abundance. Modern techniques need to be applied to measure an elusive albeit impressive phenomenon.

A great opportunity to study meteors was the occasion of the recent Leonid storms. Participating researchers from seven countries deployed a wide range of spectrographs, imagers, and resonant lidar during the four missions of the Leonid Multi-Instrument Aircraft Campaign, in 1998, 1999, 2001 and 2002. This included mid-IR and near-IR spectrographs, a sub-mm spectrometer, and highspeed imagers $(1000 \mathrm{f} / \mathrm{s})$. Spectacular showers were observed at all occasions. Each mission included the NKC-135 "FISTA" aircraft, on three occasions accompanied by a second aircraft for stereoscopic observations. FISTA offered 20 upward looking windows, $12^{\prime \prime}$ in diameter with optical quality glass and trainable eyeball assemblies for pointing instruments to meteor persistent trains. A typical mission profile would bring some 25-35 researchers to the best location for viewing the storm, at an elevation of $39,000 \mathrm{ft}$, for several hours surrounding the time of the meteor storms (Jenniskens et al. 2000b; Jenniskens \& Russell 2002).

\section{Meteor Flow Model}

In order to understand these spectroscopic studies, the first Direct Monte Carlo Simulation model of the meteoric flow field was calculated to describe the rarefied flow conditions prevalent for meteoroids at high altitude (Boyd 2000; Jenniskens et al. 2000a). In addition, the process of ablation was studied by traditional fluid dynamics (Popova et al. 2000). The emerging picture is that initial collisions with the meteoroid create a vapor cloud, with each air collision releasing up to 40 atoms or molecules from the surface of the meteoroid (Popova et al. 
2001). The vapor moves away from the surface of the meteoroid thermally until hit by an air molecule, that is out to about one mean free path in air (5-10 times the body size).

Subsequent air collisions are predominantly with the vapor cloud (Padevet 1977), causing atomization and ionization of meteoric vapor and air molecules. In this process, impact excitation leads to much of the observed optical emission of meteors (Öpik 1955, 1958). The collisional cascade expands these products into $\mathrm{a} \sim 2 \mathrm{~m}$ diameter region behind the meteoroid, about 5-10 mean free paths determined by the number of collisions that are needed to slow down the excited molecules (Jones 1995; Boyd 2000). These widths are consistent with observations of optical trail widths (Hawkins \& Whipple 1958; Cook et al. 1962; Hawkins 1963). It is from this region that most meteoric emission is observed (Fig. 1).

A significant fraction of vapor cloud mass appears to be lost by another mechanism, and that is momentum transfer in the vapor cloud. This matter remains more near the center of the meteoroids' path, where meteoric debris slowed down by direct air collisions will also be found.

During the 2001 campaign it was discovered that UV light generated in the impact excitation is absorbed by the ambient atmosphere leading to an optical glow (Stenbeak-Nielsen \& Jenniskens 2002). Intensified video meteor images taken at a rate of 1000 per second show a spherical region of light centered on the meteoroid, with a parabolic cutout. The age old question of how radar head-echo electrons can be lost from the meteor head quick enough for the radar response not to smear out into a cylindrical area is answered by the discovery of a "shock" with a large stand-off distance in the high frame-rate images, which must coincide with the loss of electrons in the spherical region.

\section{Meteor Chemistry}

Murad (2001) has pointed out that chemistry can start even before ablation occurs, in the solid state on the grain surfaces during heating,. These include solid state reactions such as transitions from silicate phases to oxide phases, evaporation of gasses, melting and loss of low-boiling point metals such as sodium and potassium. Brownlee et al. (2002) found that heating of IDP's with an electron beam tends to separate organics into soot particles enriched in metallic iron, with a silicate residue in melted droplets. The product of such processes may be identified as micrometeorites in ocean sediments, but those are often only partially melted.

It is commonly assumed that the onset of intense evaporation coincides with the meteoroid surface, or body as a whole, reaching evaporation temperatures of $\mathrm{T} \sim 2100-2800 \mathrm{~K}$, which is thought to be around $103 \mathrm{~km}$ for a $1 \mathrm{~cm}$ Leonid and $\sim 116 \mathrm{~km}$ for fainter meteors (Levin 1961; Ceplecha 1961). However, significant metal atom emissions are typically observed in Leonid meteors as high as $\sim 135$ $\mathrm{km}$, and are not necessary the result of the loss of organic matter as proposed by Elford et al. (1997). The optical spectra show no clear evidence of a sequential loss of minerals as in the differential ablation models by McNeil et al. (1998). It has been recognized that sodium is lost earlier than $\mathrm{Fe}$ and $\mathrm{Mg}$ in fragile cometary meteoroids, a process that must be linked to the fact that the more 
volatile minerals are more exposed. Not even in meteor endflares do we recognize all main elements. These observations argue in favor of a sputtering mechanism by impinging air molecules (Wood 1957) or by breaking of bonds by the intense radiation field. The evaporating atoms and molecules must quickly loose the heat from the surface and prevent melting of the meteoroids. Radiative losses are negligible for meteoroids larger than 1 micron (Jones \& Kaiser 1967).

The open question is to what level the vapor pressure builds up and whether it leads to full shielding of the meteoroid from the impinging air molecules (Öpik 1958) with perhaps even a well defined interaction layer (Rajchl 1969). If impinging air molecules continue to hit the surface, then the vapor density does not fully shield the meteoroid. Indirect collisions from the hot vapor cloud can also sputter the surface, but temperatures are only of order $4,000-40,000 \mathrm{~K}$ for a 0.1-1 cm sized body at 90-110 km altitude (Popova et al. 2000), and this will tend to result in less heat loss by evaporation and more by heating of the meteoroid. Hence, the conditions in the vapor cloud are probably those of low particle density but high radiation density. Typically, there will be only one hot collision or a few warm collisions before an atom or molecule is decelerated into the wake of the meteoroid. Most collisions with air molecules occur at the outer edges of the vapor cloud (which has the highest surface area exposed to the impinging air molecules).

The air molecule will gain translational energy from the collision relative to the ambient atmosphere and the vapor cloud atom will also gain translational energy relative to the meteoroid. Both will continue to collide with ambient air molecules until slowed down. This leads to an excitation of the ambient atmosphere and metal atoms by collisions, responsible for most of the observed light originating from a meteor. Initial collisions are violent and can lead to ions, many of which are present in meteor spectra $\left(\mathrm{N}^{+}, \mathrm{Ca}^{+}, \mathrm{Fe}^{+}, \mathrm{Si}^{+}, \ldots\right)$. A hot component has been detected in meteor spectra (Borovicka 1994), but the origin of these emissions remains unknown. A faster or larger meteoroid will lead to more violent or more collisions, resulting in a larger region of plasma. Once the air plasma formed in this manner is established, it will cool gradually.

Does this process lead to the fragmentation of organic compounds in the meteoroid? We searched for $\mathrm{CN}$ emission in our spectra that show strong metal atom line emission and found $[\mathrm{CN} / \mathrm{Fe}]<0.03$ (Jenniskens et al. 2003a). If all nitrogen in the complex organic matter of comet Halley's dust would have come off as $\mathrm{CN}$, the value would have been $[\mathrm{N}] /[\mathrm{Fe}]=0.79$. This suggests that nitrogen in the organic matter is not lost in the form of $\mathrm{CN}$. We do see a signature of hydrogen emission, but only after a catastrophic breakup of a large meteoroid. If this hydrogen can be associated with the organic content of the meteoroid, then at least some loss of functional groups are lost.

The particular temperature of $\mathrm{T} \sim 4,400 \mathrm{~K}$ in the wake of the meteor is of interest for inducing chemistry in the ambient atmosphere because it is at a temperature where $\mathrm{CO}_{2}$ does not only break up in $\mathrm{CO}+\mathrm{O}$, but $\mathrm{CO}$ breaks up in $\mathrm{C}+\mathrm{O}$. At this temperature, small amounts of reduced compounds will be formed in a $\mathrm{CO}_{2}$-rich atmosphere. In LTE conditions, much of the electron charge is balanced by $\mathrm{NO}^{+}$, while at higher temperatures much of the charge is carried by $\mathrm{C}^{+}$or $\mathrm{O}^{+}$. The NO prod'uction rate has yet to be calibrated by observations. 
We also discovered that rapid spinning of the meteoroid can eject small fragments to even larger distances, where each fragment can act as a small meteor in itself, greatly expanding the area over which air is being processed. Ejection speeds comparable to the entry speed of the meteoroid have been measured in narrow-band images of meteors in the $518 \mathrm{~nm}$ line of $\mathrm{Mg} \mathrm{I}$ (LeBlanc et al. 2000, Taylor et al. 2000). Even a fast $(72 \mathrm{~km} / \mathrm{s})$ and large $(100-1000 \mathrm{~g})$ meteoroid can deposit solid debris in its path, if conditions are favorable. Bright fireballs leave a persistent "afterglow" in a repetitive pattern with sharp dark-to-light transitions. This phenomenon is indicative of spinning modulated ablation. Strong red continuum emission associated with such breakup has been attributed to meteoric debris left in the wake of the catastrophic fragmentation (Borovicka \& Jenniskens 2000). At the same time, a continuum emission has been observed in the persistent train that might originate from meteoric debris (Russell et al. 2000). Russell et al. also made a tentative detection of the 3.4 micron emission band, which may be due to organic matter still mixed in with the silicate dust.

\section{Physical Conditions In Meteor Path}

The extended wake of debris causes a gradual cooling of the afterglow in large meteoroids. For a -12 magnitude fireball, the ablation vapor cools from $\sim 4500 \mathrm{~K}$ to $\sim 1200 \mathrm{~K}$ in a few seconds time (Borovicka \& Jenniskens 2000), while a -3 magnitude Leonid's plasma cools in about $\sim 0.1 \mathrm{~s}$. This compares to timescales of cooling of the electrons created in collisional ionization of order $0.001 \mathrm{~s}$ at $80 \mathrm{~km}$, to about $0.1 \mathrm{~s}$ at $115 \mathrm{~km}$, faster for brighter meteors (Baggaley \& Webb 1977). Persistent mid-infrared emission remains detected for longer periods of time, even when the gas and dust have cooled to just $50 \mathrm{~K}$ over the ambient $\mathrm{T} \sim 250 \mathrm{~K}$ a few minutes after the fireball (Russell et al. 2000; Chu et al. 2000a). Intensity estimates of the OI green line wake (Halliday 1959), based on the Barth mechanism (Barth \& Hildebrand 1961), show that about $15 \%$ of the $\mathrm{O}_{2}$ in the initial train is dissociated in a -12 magnitude Leonid (Jenniskens et al. 2000c). A low level of ionization remains present for long periods of time, responsible for VHF and UHF radar reflections. Strong recombination line emission was identified recently in the wake of the meteors over a longer timescale, especially in bright Leonid fireballs for periods of several tens of seconds. The ionization efficiency per gram varies with entry speed (Whipple 1955), making this a more dominant phenomenon in fast Leonid meteors. This emission is very different from that of the meteor itself or its afterglow. The recombination of metal ions and free electrons was previously discarded as a source of meteor train emission (Cook \& Hawkins 1956), although Baggaley (1977) invoked air plasma ion-ion recombination as a likely mechanism.

Over even longer timescales, a persistent train is observed that can last longer than 1 hour. The train spectrum shows sodium emission lines and a broad molecular band continuum, now identified as the $\mathrm{FeO}$ orange arc band (Jenniskens et al. 2000c). The luminous mechanism is thought to be the catalytic recombination of ambient ozone with oxygen atoms in the trail through the Chapman airglow mechanism (Chapman 1939; Baggaley 1975, 1976). Indeed, iron atoms are found to be present in the path of relatively faint meteors over timescales of half an hour in resonant lidar studies (Chu et al. 2000b). 
Often, only one of several elements is detected at any given position in an atom debris trail with resonant lidar (Von Zahn et al. 1999), who have ascribed this to differential ablation. However, the expected altitude dependence of ablation of less volatile minerals is not observed and we do not see such differential ablation in optical spectra. Rather, this suggests that various forms of chemistry will occur in the neutral atom debris trails over timescales of several minutes or hours between deposition and detection. It is unclear if that also affects the organic molecules in the trail.

The existence of $\mathrm{nm}$-sized meteoric recondensed vapor in trains remains unproven. Kelley et al. (1998) gave indirect evidence from a sudden brief change in ion density measured during a rocket flight. Efforts to detect such dust with strong lasers have not yet been successful (Kelley et al. 2000).

Acknowledgments. Research on the 2001 Leonid MAC mission was jointly supported by NASA's Astrobiology \& Planetary Astronomy programs. The FISTA aircraft is operated by the USAF $418^{\text {th }}$ Flight Test Squadron at Edwards AFB.

\section{References}

Baggaley, W. J. 1975, Nature, 257, 567

Baggaley, W. J. 1976, Bull. Astron. Inst. Czechoslov., 27, 296

Baggaley, W. J. 1977, Bull. Astron. Inst. Czechoslov., 28, 108

Baggaley, W. J., \& Webb, T. H. 1977, J. of Atmosp. and Ter. Phys., 39, 1399

Barth, C. A., \& Hildebrandt, A. F. 1961, J. of Geoph. Res., 66, 985

Borovicka, J. 1994, Space Sci., 42, 145

Borovicka, J., \& Jenniskens ,P. 2000, Earth, Moon and Planets, 82-83, 399

Boyd, I. D. 2000, Earth, Moon and Planets 82-83, 93

Bronshten, V. A. 1983, Phys. of Meteoric Phenomena, (Dordrecht Reidel), 356

Brownlee, D. E., Joswiak, D. J., Kress, M. E., Taylor, S., \& Bradley, J. 2002, in 33rd Annual Lunar and Planetary Science Conference, (Houston), 1786

Ceplecha, Z. 1961, Bull. Astron. Inst. Czechoslov., 12, 191

Ceplecha, Z., Borovicka, J., Elford, W. G., ReVelle, D. O., Hawkes, R. L., Porubcan, V., \& Simek, M. 1998, Space Science Reviews, 84, 327

Chapman, S. 1939, ApJ, 90, 309

Chyba, C. F., \& Sagan, C. 1992, Nature, 355, 125

Chyba, C. F., \& Sagan, C. 1997, in Comets and the Origin and Evolution of Life, ed. P.J. Thomas, C.F. Chyba, C.P. McKay, (Springer Verlag), 147

Chu, X., Liu, A. Z., Papen, G., Gardner, C. S., Kelley, M. C., Drummond, J., \& Fugate, R. 2000a, Geophys. Res. Lett., 27, 1815

Chu, X., Pan, W., Papen, G., Gardner, C. S., \& Swenson, G. 2000b, Geophys. Res. Lett., 27, 1807

Cook, A. F., \& Hawkins, G. S. 1956, AJ, 61, 174

Cook, A. F., Hawkins, G. S., \& Stienon, F. M. 1962, AJ, 67, 158

Elford, W. G., Steel, D. I., \& Taylor, A. D. 1997, Adv. Space Res., 20, 1501 
Halliday, I. 1959, Contr. Dominion Obs., Ottawa, Ontario, 2, 441

Hawkins, G. S., \& Whipple, F. L. 1958, Harvard Reprint Ser. II, 138, 283

Hawkins, G. S., 1963, Smiths. Contr. Astrophys., 7, 23

Jenniskens, P. 2001, in Proceedings of the Meteoroids, Swedish Institute of Space Physics, 247

Jenniskens, P., \& Russell, R. W. 2002, ISAS SP-16 (in press)

Jenniskens, P., Wilson, M. A., Packan, D., Laux, C. O., Boyd, I.D., Popova, O., \& Fonda, M. 2000a, Earth, Moon and Planets, 82-83, 57

Jenniskens, P., Butow, S., \& Fonda, M. 2000b, Earth, Moon and Planets, 82, 1

Jenniskens, P., Nugent, D., \& Plane, J. M. C. 2000c, Earth, Moon and Planets, 82,471

Jenniskens, P., Schaller, E. L., Laux, C. O., Schmidt, G., \& Rairden, R. L. 2003a, Astrobiology (submitted)

Jones, J., \& Kaise,r T. R. 1967, MNRAS, 133, 411

Jones, W. 1995, MNRAS, 275, 812

Kelley, M. C., Alcala, C., \& Cho, J. Y. N. 1998, J. Atmosph. and Solar-Terr. Phys., 60, 359

Kelley, M. C., Gardner, C., Drummond, J., Armstrong, T., Liu, A., Chu, X., Papen, G., Kruschwitz, C., Loughmiller, P., Grime, B., \& Engleman, J. 2000, Geophys. Res. Lett., 27, 1811

Le Blanc, A. G., Murray, I. S., Hawkes, R. L., Worden, P., Campbell, M. D., Brown, P., Jenniskens, P., Correll, R. R., Montague, T., \& Babcock, D. D. 2000, MNRAS, 313, L9

Levin, B. Yu. 1961, Physikalische Theorie der Meteore und die meteoritische Substanz im Sonnensystem, Teil II. Scientia Astronomica 4, (AkademieVerliag: Berlin), 330

McNeil, W. J., Lai, S. T., \& Murad, E. 1998, J. Geophys. Res., 103, 10899

McKinley, D. W. R. 1961, Meteor Science and Engineering. (New York: McGrawHill Book Company Inc.), 309

Murad, E. 2001, in Proceedings of the Meteoroids, Swedish Institute of Space Physics, 229

Öpik, E. J. 1955, J. Atmosph. and Terrestrial Phys., 2, 29

Öpik, E. J. 1958, Physics of meteor flight in the atmopshere, (New York: Interscience Publishing)

Oró, J. 1961, Nature 190, 389

Padevet, V. 1977, Bull. Astron. Inst. Czechoslov., 28, 90

Popova, O. P., Sidneva, S. N., Shuvalov, V. V., \& Strelkov, A. S. 2000, Earth, Moon and Planets 82-83, 109

Popova, O.P., Sidneva, S. N., Strelkov, A. S., \& Shuvalov, V. V. 2001, in Proceedings of the Meteoroids, Swedish Institute of Space Physics, 237

Rajchl, J. 1969. Bull. Astron. Inst. Czechoslov., 20, 363

Russell, R. W., Rossano, G. S., Chatelain, M. A., Lynch, D. K., Tessensohn, T. K., Abendroth, E., \& Kim, D. 2000, Earth, Moon and Planets, 82, 439 
Stenbaek-Nielsen, \& Jenniskens, P. 2002, Leonid at 1000 Frames per Second. ISAS SP-16 (in press)

Taylor, M. J., Gardner, L. C., Murray, I. S., \& Jenniskens, P. 2000, Earth, Moon and Planets, 82-83, 379

Von Zahn, U., Gerding, M., Höffner, J., McNeil, W. J., \& Murad E. 1999, Meteoritics Planet. Sci., 34, 1017

Whipple, F. L. 1955, ApJ, 121, 241

Wood, J. A. 1957, AJ, 62, 40

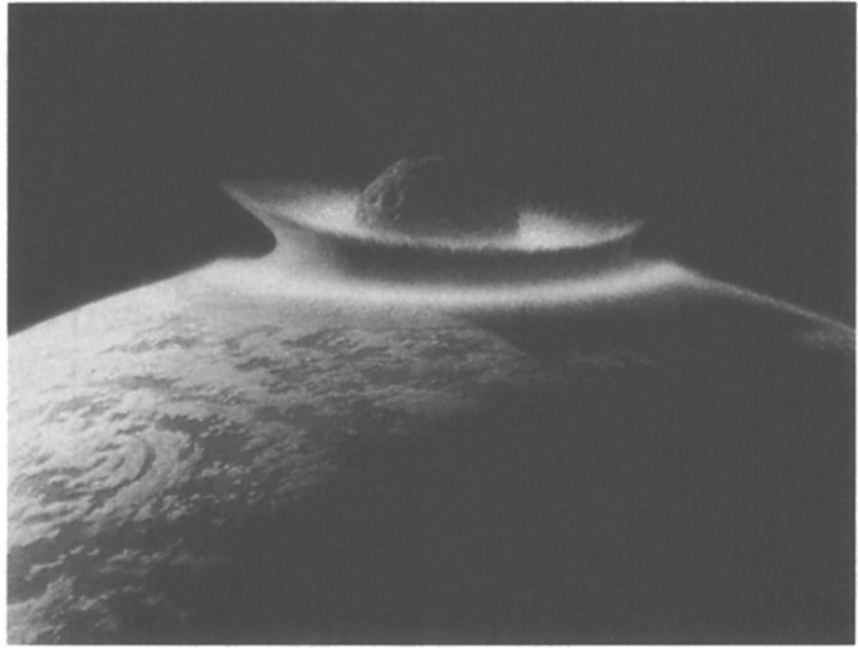

(photo: NASA) 